\title{
Postpartum attacks of acute-onset chronic inflammatory demyelinating polyneuropathy in a young woman with successive pregnancies
}

\author{
Woojun Kim ${ }^{1}$, Jean Hee Kim ${ }^{1}$, Dae Woong Bae ${ }^{2}$, Jae Young $\mathrm{An}^{2}$ \\ ${ }^{1}$ Department of Neurology, Seoul St. Mary's Hospital, College of Medicine, The Catholic University of Korea, Seoul, Republic of Korea; \\ ${ }^{2}$ Department of Neurology, St. Vincent's Hospital, College of Medicine, The Catholic University of Korea, Seoul, Republic of Korea
}

Correspondence to: Jae Young An. Department of Neurology, St. Vincent's Hospital, The Catholic University of Korea, 93-6, Ji-dong, Paldal-gu, Suwon, Gyeonggi-do, 442-723, Korea. Email: nrjyan@gmail.com.

Submitted Feb 11, 2021. Accepted for publication May 19, 2021.

doi: $10.21037 /$ qims-21-177

View this article at: http://dx.doi.org/10.21037/qims-21-177

\section{Introduction}

Chronic inflammatory demyelinating polyneuropathy (CIDP) is a clinically heterogenous, immune-mediated neuropathy with a relapsing or progressive course lasting more than 8 weeks. However, some patients with CIDP have an acute onset, similar to Guillain-Barré syndrome (GBS), but subsequently develop a chronic or relapsing course (1). These are considered as acute-onset CIDP (A-CIDP). However, definite electrophysiological findings that can differentiate A-CIDP from GBS are lacking, and thus it might be difficult to differentiate A-CIDP from GBS in many cases $(2,3)$. Unlike other autoimmune diseases, CIDP usually presents in older adults, with a peak incidence between the ages of 40 and 60 years, and it more frequently occurs in men than in women (4). There is no clear understanding about the background mechanism underlying this gender-related difference.

Here, we describe the serial electrophysiological and ultrasonographic findings of a 22-year-old woman with A-CIDP that developed in successive postpartum periods.

\section{Case presentation}

A previously healthy 22 -year-old woman presented with ascending weakness and distal paresthesia for 3 weeks. Five weeks before admission, she had given birth vaginally to her first baby at full term without any complications. Three days later, she received Tdap (tetanus-diphtheria- acellular pertussis) vaccine, and the weakness began 10 days after vaccination. A neurological examination showed weakness of the upper [Medical Research Council (MRC), 4/5] and lower extremities (MRC, 3/5), numbness in the distal extremities, and reduced deep tendon reflexes. The weakness progressed in the upper extremities (MRC 3/5) and lower extremities (MRC 2/5) over 4 days after admission. Cerebrospinal fluid analysis revealed only protein elevation $(164 \mathrm{mg} / \mathrm{dL})$. A nerve conduction study (NCS) showed diffuse demyelinating sensory motor polyneuropathy with conduction block, absent F-waves, and absent sensory potentials of the median and ulnar nerves, but the sural nerve was preserved (Figure 1A). High-resolution ultrasound (HRUS) with a linear array probe of 6-18 MHz revealed significant swelling of the peripheral nerves, including the brachial plexus, median, ulnar, radial, tibial, and peroneal nerves, evaluated based on increased cross-sectional area (CSA) (Figure 1A). There was no swelling of the vagus and sural nerves. Anti-myelinassociated glycoprotein antibody and anti-ganglioside antibodies, including anti-GM1 and anti-Gb1b, and GQ1b were negative. Intravenous high-dose immunoglobulin $(0.4 \mathrm{~g} / \mathrm{kg} /$ day for 5 days) was started with diagnosis of GBS. After 10 days, weakness began to improve, and only mild paresthesia remained after 2 months. A 12-month followup NCS showed improved demyelinating pathology, and follow-up HRUS revealed mildly reduced CSA (Figure 1B). Because she was preparing for her next pregnancy, we did not provide treatment, despite considering CIDP as the 

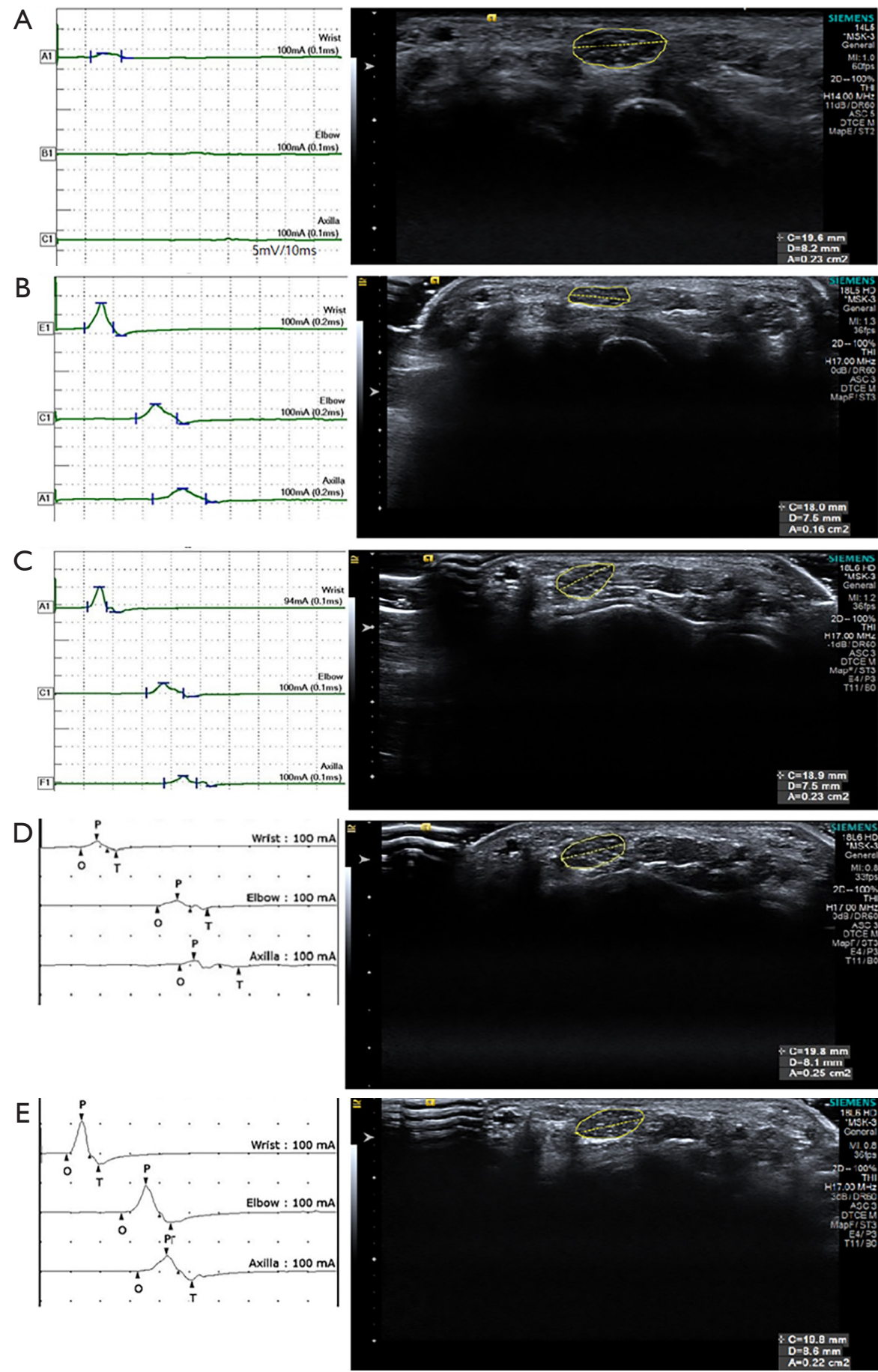

Figure 1 Serial motor nerve conduction studies (NCS) and ultrasound (US) of the right median nerve. (A) Initial studies on the patient's first attack show increased distal motor latency $(11.8 \mathrm{~ms})$ and increased cross-sectional area (CSA: $\left.23 \mathrm{~mm}^{2}\right)$ of the median nerve at the wrist. (B) 12-month follow-up NCS and US show improved demyelinating neuropathies and reduced CSA (16 mm²). (C) 19-month follow-up studies (22nd week of her second gestation) reveal mild aggravation in NCS and more increased CSA (23 mm ${ }^{2}$ ) without recurrence of symptoms. (D) Follow-up studies at the second attack during her second postpartum show a deterioration in NCS and persistent increased CSA $\left(25 \mathrm{~mm}^{2}\right)$. (E) Two months after steroid treatment, follow-up studies show marked improvement in NCS, but swelling of the nerve is slightly reduced (CSA: $22 \mathrm{~mm}^{2}$ ). The reference value of the median nerve CSA at the wrist in our laboratory is $13 \mathrm{~mm}^{2}$. 
cause of neuropathy. A 19-month follow-up study (22nd week of her second gestation) revealed mild aggravation in NCS and more increased CSA, without any change of symptoms (Figure 1C). Ten days after the second delivery, she complained of mild weakness of all four extremities and aggravated distal paresthesia, which had been getting worse (MRC, 4/5) for a week. She denied a history of preceding infection or vaccination. NCS and HRUS revealed aggravated conduction block and swelling of the nerves (Figure 1D). She was treated with intravenous methylprednisolone (1,000 $\mathrm{mg}$ for 3 days) followed by oral prednisolone for maintenance therapy. Weakness improved markedly within 2 weeks. Follow-up studies, performed 2 months after her second relapse, showed marked improvement in NCS, but swelling of the nerve was slightly reduced (Figure 1E). All procedures performed in studies involving human participants were in accordance with the ethical standards of the Catholic University of Korea St. Vincent's Hospital Institutional Review Board and with the Helsinki Declaration (as revised in 2013). Written informed consent was obtained from the patient.

\section{Discussion}

In one retrospective study of nine women with CIDP who became pregnant, the greatest risk of exacerbation of symptoms occurred in the third trimester and in the postpartum period (1). It is well known that the immune system changes dramatically during pregnancy, and so many autoimmune diseases go into remission, only to flare again in the early postpartum period. Typically, T helper type 1 (Th1) and T helper type 17 autoimmune disorders, such as myasthenia gravis and multiple sclerosis, improve as a result of the rise in T helper type 2 (Th2) cytokines in pregnancy, whereas Th2-type autoimmune disorders, such as systemic lupus erythematosus, may deteriorate in pregnancy (5). After delivery, the immune system returns to a non-pregnant state. A shift towards Th1 dominance, and a fall in Th2 and regulatory $\mathrm{T}$ cells, followed by altered cytokine pattern in the first weeks following delivery have been reported (6,7). Mei et al. (8) found upregulation of Th1 and downregulation of Th2-type cytokines in CIDP patients and upregulation of some Th2-type cytokines in patients with vasculitic neuropathy. This immunological shift in the postpartum period may have contributed to the pathogenesis of successive relapses in our patient.

Differentiating A-CIDP from GBS is important, because there have different treatment strategies and outcomes.
Addition of nerve ultrasound could shorten the time to diagnosis and could possibly improve identification of CIDP (9). Nerve enlargement is the most intriguing finding in immune mediated neuropathies in ultrasound, in acute as well as in chronic types (10). A previous study showed that ultrasonic sensory sparing in combination with enlarged roots and vagus is the hallmark finding in GBS, whereas in A-CIDP, enlarged sensory and multifocally enlarged sensorimotor nerves are key differentiation features to GBS in the early stage (2). A 6-month follow-up ultrasound showed that the significant nerve enlargement persisted in CIDP, whereas, nerve enlargement almost normalized in GBS (2). In our patient, there was widespread nerve enlargement except for the vagus and sural nerves, in the acute period, and these enlargements tended to decrease, but were not normalized in follow-up studies. Furthermore, the CSA was increased during the second pregnancy without change of symptoms and more increased in second relapse after delivery. Follow-up ultrasound should be considered when ultrasonic sural sparing and enlargement of roots are simultaneously found in the initial ultrasound.

Although a definitive conclusion cannot be drawn from a single case report, physicians should consider the possibility of A-CIDP in patients who develop acute inflammatory demyelinating polyneuropathy during the postpartum period, and serial evaluations of HRUS in addition to NCS might be needed for distinguishing CIDP from GBS and for predicting relapse of CIDP.

\section{Acknowledgments}

Funding: None.

\section{Footnote}

Conflicts of Interest: All authors have completed the ICMJE uniform disclosure form (available at http://dx.doi. org/10.21037/qims-21-177). The authors have no conflicts of interest to declare.

Ethical Statement: The authors are accountable for all aspects of the work in ensuring that questions related to the accuracy or integrity of any part of the work are appropriately investigated and resolved. All procedures performed in studies involving human participants were in accordance with the ethical standards of the Catholic University of Korea St. Vincent's Hospital Institutional Review Board and with the Helsinki Declaration (as revised 
in 2013). Written informed consent was obtained from the patient.

Open Access Statement: This is an Open Access article distributed in accordance with the Creative Commons Attribution-NonCommercial-NoDerivs 4.0 International License (CC BY-NC-ND 4.0), which permits the noncommercial replication and distribution of the article with the strict proviso that no changes or edits are made and the original work is properly cited (including links to both the formal publication through the relevant DOI and the license). See: https://creativecommons.org/licenses/by-nc-nd/4.0/.

\section{References}

1. McCombe PA, Pollard JD, McLeod JG. Chronic inflammatory demyelinating polyradiculoneuropathy. A clinical and electrophysiological study of 92 cases. Brain 1987;110:1617-30.

2. Grimm A, Oertl H, Auffenberg E, Schubert V, Ruschil C, Axer H, Winter N. Differentiation Between GuillainBarré Syndrome and Acute-Onset Chronic Inflammatory Demyelinating Polyradiculoneuritis-a Prospective Followup Study Using Ultrasound and Neurophysiological Measurements. Neurotherapeutics 2019;16:838-47.

3. Dionne A, Nicolle MW, Hahn AF. Clinical and electrophysiological parameters distinguishing acute-onset chronic inflammatory demyelinating polyneuropathy from acute inflammatory demyelinating polyneuropathy. Muscle Nerve 2010;41:202-7.

Cite this article as: Kim W, Kim JH, Bae DW, An JY. Postpartum attacks of acute-onset chronic inflammatory demyelinating polyneuropathy in a young woman with successive pregnancies. Quant Imaging Med Surg 2021;11(11):4683-4686. doi: 10.21037/qims-21-177
4. Rodríguez Y, Vatti N, Ramírez-Santana C, Chang C, Mancera-Páez O, Gershwin ME, Anaya JM. Chronic inflammatory demyelinating polyneuropathy as an autoimmune disease. J Autoimmun 2019;102:8-37.

5. Bränn E, Edvinsson $\AA$, Rostedt Punga A, SundströmPoromaa I, Skalkidou A. Inflammatory and antiinflammatory markers in plasma: from late pregnancy to early postpartum. Sci Rep 2019;9:1863.

6. La Rocca C, Carbone F, Longobardi S, Matarese G. The immunology of pregnancy: regulatory $\mathrm{T}$ cells control maternal immune tolerance toward the fetus. Immunol Lett 2014;162:41-8.

7. Sato T, Terasawa Y, Higa H, Matsuno H, Arai A, Omoto S, Mitsumura H, Toyoda C, Koike H, Iguchi Y. Nerve Ultrasound, Electrophysiological, and Clinical Changes in Treatment-Naive Chronic Inflammatory Demyelinating Polyneuropathy: A Case Report. J Clin Neurophysiol 2019;36:82-4

8. Mei FJ, Ishizu T, Murai H, Osoegawa M, Minohara M, Zhang KN, Kira J. Th1 shift in CIDP versus Th2 shift in vasculitic neuropathy in CSF. J Neurol Sci 2005;228:75-85.

9. Herraets IJT, Goedee HS, Telleman JA, van Eijk RPA, van Asseldonk JT, Visser LH, van den Berg LH, van der Pol WL. Nerve ultrasound improves detection of treatmentresponsive chronic inflammatory neuropathies. Neurology 2020;94:e1470-9.

10. Zaidman CM, Harms MB, Pestronk A. Ultrasound of inherited vs. acquired demyelinating polyneuropathies. J Neurol 2013;260:3115-21. 